\title{
VON NEUMANN'S INEQUALITY FOR COMMUTING, DIAGONALIZABLE CONTRACTIONS. II
}

\author{
B. A. LOTTO AND T. STEGER
}

(Communicated by Theodore W. Gamelin)

\begin{abstract}
We construct a triple $T=\left(T_{1}, T_{2}, T_{3}\right)$ of commuting, diagonalizable contractions on $\mathbf{C}^{5}$ and a polynomial $p$ in three variables for which $\|p(T)\|>\|p\|_{\infty}$, where $\|p\|_{\infty}$ denotes the supremum norm of $p$ over the unit polydisk in $\mathbf{C}^{3}$.
\end{abstract}

\section{INTRODUCTION}

In part I [2], the first author showed that von Neumann's inequality

$$
\|p(T)\| \leq\|p\|_{\infty}
$$

holds for all polynomials $p$ in $n$ variables, where $T$ is an $n$-tuple of commuting, diagonalizable contractions on $\mathbf{C}^{N}$ that satisfies some additional hypotheses. Here $\|p(T)\|$ denotes the operator norm of $p(T)$ and $\|p\|_{\infty}$ denotes the supremum norm of $p$ over the unit polydisk of $\mathbf{C}^{n}$. In the present work we present an example to show that the extra hypotheses cannot be removed. Our example is based on an example due to Kaijser and Varopoulos [3, addendum] that shows that (1) can fail with $n=3$ and $N=5$. This example consists of nilpotents; our example is obtained by perturbing their example to diagonalizables.

\section{THE COUNTEREXAMPLE}

Theorem. There are thiee commuting, diagonalizable contractions $T_{1}, T_{2}$, and $T_{3}$ on $\mathbf{C}^{5}$ and a polynomial $p$ in three variables such that $\left\|p\left(T_{1}, T_{2}, T_{3}\right)\right\|$ $>\|p\|_{\infty}$.

We start with the example of Kaijser and Varopoulos mentioned above. Use the standard inner product on $\mathbf{C}^{5}$. The three operators

$$
A_{1}=\left(\begin{array}{ccccc}
0 & 0 & 0 & 0 & 0 \\
1 & 0 & 0 & 0 & 0 \\
0 & 0 & 0 & 0 & 0 \\
0 & 0 & 0 & 0 & 0 \\
0 & 1 / \sqrt{3} & -1 / \sqrt{3} & -1 / \sqrt{3} & 0
\end{array}\right),
$$

Received by the editors November 1, 1991 and, in revised form, July 1, 1992; presented at AMS meeting \#876, Dayton, Ohio, October 1992.

1991 Mathematics Subject Classification. Primary 47A63; Secondary 15 A60. 


$$
A_{2}=\left(\begin{array}{ccccc}
0 & 0 & 0 & 0 & 0 \\
0 & 0 & 0 & 0 & 0 \\
1 & 0 & 0 & 0 & 0 \\
0 & 0 & 0 & 0 & 0 \\
0 & -1 / \sqrt{3} & 1 / \sqrt{3} & -1 / \sqrt{3} & 0
\end{array}\right),
$$

and

$$
A_{3}=\left(\begin{array}{ccccc}
0 & 0 & 0 & 0 & 0 \\
0 & 0 & 0 & 0 & 0 \\
0 & 0 & 0 & 0 & 0 \\
1 & 0 & 0 & 0 & 0 \\
0 & -1 / \sqrt{3} & -1 / \sqrt{3} & 1 / \sqrt{3} & 0
\end{array}\right)
$$

on $\mathrm{C}^{5}$ are commuting contractions, and the polynomial

$$
p\left(z_{1}, z_{2}, z_{3}\right)=z_{1}^{2}+z_{2}^{2}+z_{3}^{2}-2 z_{1} z_{2}-2 z_{1} z_{3}-2 z_{2} z_{3}
$$

satisfies $\|p\|_{\infty}=5$ and $\left\|p\left(A_{1}, A_{2}, A_{3}\right)\right\|>5$. We will produce, for every $\epsilon>$ 0 , perturbations $A_{j}^{(\epsilon)}$ of $A_{j}(j=1,2,3)$ that commute and are diagonalizable, with the additional property that $A_{j}^{(\epsilon)} \rightarrow A_{j}$ as $\epsilon \rightarrow 0$. Since then $\left\|A_{j}^{(\epsilon)}\right\| \rightarrow$ $\left\|A_{j}\right\|=1$ as $\epsilon \rightarrow 0$, we may replace $A_{j}^{(\epsilon)}$ by $A_{j}^{(\epsilon)} /\left\|A_{j}^{(\epsilon)}\right\|$ and assume that $A_{j}^{(\epsilon)}$ is a contraction. We will then have

$$
\left\|p\left(A_{1}^{(\epsilon)}, A_{2}^{(\epsilon)}, A_{3}^{(\epsilon)}\right)\right\| \rightarrow\left\|p\left(A_{1}, A_{2}, A_{3}\right)\right\|>5
$$

as $\epsilon \rightarrow 0$, so that for small enough $\epsilon$ we have $\left\|p\left(A_{1}^{(\epsilon)}, A_{2}^{(\epsilon)}, A_{3}^{(\epsilon)}\right)\right\|>5$. Setting $T_{j}=A_{j}^{(\epsilon)}$ for such an $\epsilon$ gives the theorem. We therefore need only construct the perturbations.

Let

$$
X=\left(\begin{array}{rrrrr}
0 & 0 & 0 & 0 & 1 \\
0 & 1 & -1 & -1 & 0 \\
0 & -1 & 1 & -1 & 0 \\
0 & -1 & -1 & 1 & 0 \\
1 & 0 & 0 & 0 & 0
\end{array}\right)
$$

and let $Y$ be any matrix such that $Y^{-1}=Y^{\mathrm{t}} X$, that is, such that $Y Y^{\mathrm{t}}=X^{-1}$. We can produce one such $Y$ as follows. Since $X^{-1}$ is real symmetric, there is a real orthogonal matrix $U$ and a diagonal matrix $D$ such that $X^{-1}=U D U^{\mathrm{t}}$. Let $\sqrt{D}$ be a diagonal matrix whose square is $D$ and set $Y=U \sqrt{D} U^{\mathrm{t}}$.

By replacing $Y$ by $Y O$, where $O$ is a suitably chosen orthogonal matrix, we may assume that the fifth row of $Y$ contains only nonzero entries. We denote the fifth row by $y$ and think of it as an element of $\mathbf{C}^{5}$.

Consider the linear map $L$ from $C^{5}$ into operators on $C^{5}$ defined by $L a=$ $Y A Y^{\mathrm{t}}$, where $A$ is the diagonal matrix whose diagonal is $a$. The fifth row of $L a$ is $(y * a) Y^{\mathrm{t}}$, where $*$ denotes coordinatewise multiplication. Since $Y$ is invertible and $y$ has only nonzero entries, it follows that the linear map that sends $a$ to the fifth row of $L a$ is invertible. Hence, we can find $u_{1}, u_{2}$, and $u_{3}$ in $C^{5}$ such that the fifth rows of $L u_{1}, L u_{2}$, and $L u_{3}$ are $(0,1,0,0,0)$, 
$(0,0,1,0,0)$, and $(0,0,0,1,0)$, respectively. Since $L a$ is always symmetric, we have

$$
\begin{aligned}
L u_{1} & =\left(\begin{array}{lllll}
* & * & * & * & 0 \\
* & * & * & * & 1 \\
* & * & * & * & 0 \\
* & * & * & * & 0 \\
0 & 1 & 0 & 0 & 0
\end{array}\right), \\
L u_{2} & =\left(\begin{array}{lllll}
* & * & * & * & 0 \\
* & * & * & * & 0 \\
* & * & * & * & 1 \\
* & * & * & * & 0 \\
0 & 0 & 1 & 0 & 0
\end{array}\right),
\end{aligned}
$$

and

$$
L u_{3}=\left(\begin{array}{lllll}
* & * & * & * & 0 \\
* & * & * & * & 0 \\
* & * & * & * & 0 \\
* & * & * & * & 1 \\
0 & 0 & 0 & 1 & 0
\end{array}\right)
$$

For $\epsilon>0$ and $j=1,2,3$, let $U_{j}^{(\epsilon)}$ denote the diagonal matrix whose diagonal is $\epsilon u_{j}$. We have

$$
Y U_{1}^{(\epsilon)} Y^{-1}=Y U_{1}^{(\epsilon)} Y^{\mathrm{t}} X=\epsilon\left(L u_{1}\right) X=\left(\begin{array}{ccccc}
0 & * & * & * & * \\
\epsilon & * & * & * & * \\
0 & * & * & * & * \\
0 & * & * & * & * \\
0 & \epsilon & -\epsilon & -\epsilon & 0
\end{array}\right),
$$

where the missing entries are constant multiples of $\epsilon$. Let $D_{\epsilon}$ be the diagonal matrix with diagonal entries $\left(1, \epsilon^{-1}, \epsilon^{-1}, \epsilon^{-1}, \epsilon^{-2} / \sqrt{3}\right)$. Then

$$
\left(D_{\epsilon} Y\right) U_{1}^{(\epsilon)}\left(Y^{-1} D_{\epsilon}^{-1}\right)=\left(\begin{array}{ccccc}
0 & * & * & * & * \\
1 & * & * & * & * \\
0 & * & * & * & * \\
0 & * & * & * & * \\
0 & 1 / \sqrt{3} & -1 / \sqrt{3} & -1 / \sqrt{3} & 0
\end{array}\right),
$$

where the missing entries are constant multiples of $\epsilon, \epsilon^{2}$, or $\epsilon^{3}$. This is the perturbation $A_{1}^{(\epsilon)}$. Letting $Z_{\epsilon}=D_{\epsilon} Y$, we similarly set

$$
A_{2}^{(\epsilon)}=Z_{\epsilon} U_{2}^{(\epsilon)} Z_{\epsilon}^{-1}=\left(\begin{array}{ccccc}
0 & * & * & * & * \\
0 & * & * & * & * \\
1 & * & * & * & * \\
0 & * & * & * & * \\
0 & -1 / \sqrt{3} & 1 / \sqrt{3} & -1 / \sqrt{3} & 0
\end{array}\right)
$$


and

$$
A_{3}^{(\epsilon)}=Z_{\epsilon} U_{3}^{(\epsilon)} Z_{\epsilon}^{-1}=\left(\begin{array}{ccccc}
0 & * & * & * & * \\
0 & * & * & * & * \\
0 & * & * & * & * \\
1 & * & * & * & * \\
0 & -1 / \sqrt{3} & -1 / \sqrt{3} & 1 / \sqrt{3} & 0
\end{array}\right)
$$

Clearly, $A_{1}^{(\epsilon)}, A_{2}^{(\epsilon)}$, and $A_{3}^{(\epsilon)}$ commute and are diagonalizable. The missing entries of (3) and (4), like those of (2), are constant multiples of $\epsilon, \epsilon^{2}$, and $\epsilon^{3}$, so $A_{j}^{(\epsilon)} \rightarrow A_{j}$ as $\epsilon \rightarrow 0$ for $j=1,2,3$.

We have found our perturbations and the theorem is proved.

\section{QUESTIONS}

The above proof involves perturbing a triple of commuting matrices to commuting, diagonalizable ones.

Question 1. Can any triple of commuting operators on a finite-dimensional space be perturbed to become commuting and diagonalizable?

It is known that any pair of commuting matrices can be perturbed to commuting diagonalizables [1]. The natural generalization to four matrices fails, as the following example shows.

Let $\left\{e_{1}, \ldots, e_{n}\right\}$ be the standard basis for $\mathbf{C}^{n}$. We define $n$ by $n$ matrices that act as follows:

$$
\begin{gathered}
T_{1} e_{j}=e_{j+1} \quad \text { for } 1 \leq j \leq n-3, \\
T_{2} e_{n-1}=e_{n}, \quad T_{3} e_{1}=e_{n}, \quad T_{4} e_{n-1}=e_{n-2},
\end{gathered}
$$

where $T_{k} e_{j}=0$ in all other cases. The product of two distinct $T_{k}$ is zero, so these matrices commute. The algebra with identity generated by the $T_{k}$ 's has $\left(T_{1}^{j}\right)_{j=0}^{n-3}, T_{2}, T_{3}, T_{4}$ as a basis and so has dimension $n+1$. If we could perturb the $T_{k}$ 's to commuting diagonalizables, the algebra generated by the perturbations would be an $(n+1)$-dimensional commutative algebra of diagonalizable $n$ by $n$ matrices. But no such algebra exists.

This argument by dimension suggests the following questions.

Question 2. Consider a subalgebra of the $n$ by $n$ matrices, commutative and with identity. If this subalgebra has dimension no greater than $n$, can it be perturbed to a commutative subalgebra of diagonal matrices?

and

Question 3. Can a triple of commuting $n$ by $n$ matrices generate an algebra with identity of dimension greater than $n$ ?

Question 2 also suggests stabilizing an arbitrary collection of commuting $n$ by $n$ matrices.

Question 4. Given a finite collection of commuting matrices, can their direct sums with a large enough zero matrix be perturbed to commuting diagonalizables? 


\section{REFERENCES}

1. J. A. R. Holbrook, Polynomials in a matrix and its commutant, Linear Algebra Appl. 48 (1982), 293-301.

2. B. A. Lotto, Von Neumann's inequality for commuting, diagonalizable contractions. I, Proc. Amer. Math. Soc. 120 (1994), 889-895.

3. N. Th. Varopoulos, On an inequality of von Neumann and an application of the metric theory of tensor products to operator theory, J. Funct. Anal. 16 (1974), 83-100.

Department of Mathematics, University of California, Berkeley, California 94720 E-mail address: lotto@math.berkeley.edu

Current address: Department of Mathematics, Vassar College, Poughkeepsie, New York, 12601

E-mail address: BeLottoevassar.edu

Department of Mathematics, University of Chicago, Chicago, Illinois 60637

E-mail address: steger@zaphod.uchicago.edu

Current address: Department of Mathematics, University of Georgia, Boyd Graduate Studies, Athens, Georgia 30602-7403

E-mail address: steger $₫ j o e . m a t h . u g a . e d u$ 